Ann. Sci. forest., I976, 33 (I), I-I7.

\title{
LA DENSITÉ DU BOIS DE HETRE DANS LE NORD-EST DE LA FRANCE. INFLUENCE DES CARACTÉRISTIQUES DU MILIEU ET DU TYPE DE SYLVICULTURE
}

\author{
R. KELLER, F. LE TACON* et J. TIMBAL** \\ avec la collaboration technique de I). BOUchard*, G. DOUCET** et Ch. KHAFER** \\ Station de Recherches sur la Qualité des Bois, \\ * Station de Recherches sur les Sols forestiers et la Fertilisation, \\ ** Laboratoire de Phyto-écologie forestière, \\ Centre national de Recherches forestières, I. N. R. A., \\ Champenoux, 54280 Seichamps
}

\section{RÉSUMÉ}

Cette étude a été réalisée sur I 20 placettes de futaie de Hêtres, d’âge variable, du Nord-Est de la France. Ces placettes ont été choisies de manière à représenter toute la variété des conditions écologiques de cette région. Dans chacune de ces placettes, sur 30 arbres, on a mesuré le couple de torsion et on a prélevé une carotte dont on a mesuré la largeur des derniers accroissements et la densité. Les coefficients de corrélation ont ensuite été calculés entre ces différentes valeurs.

La liaison entre la densité et le couple de torsion est bonne pour les mesures effectuées à une même époque de l'année. Par contre, il n'y a pas de liaison entre la densité et la largeur des accroissements annuels. La densité diminue aussi avec le diamètre des tiges et la dimension des houppiers.

Il y a un effet stationnel très net sur la densité du bois de Hêtre. Tous les facteurs écologiques qui diminuent la fertilité des stations, diminuent également la densité.

L'effet sylviculture est plus délicat à interpréter. En effet, les corrélations calculées ne sont pas significatives et les peuplements échantillonnés ne semblent pas avoir été suffisamment éclaircis pour qu'un effet puisse être mis en évidence.

\section{I. - INTRODUCTION}

I a qualité du bois de Hêtre présente une grande variabilité, aussi bien d'une région à l'autre, qu'à l'intérieur d'une même région, ou bien à l'intérieur d'une même forêt ou d'un même peuplement.

Polge, KELIER et Thiercelin (I972) ont montré sur de jeunes plants que 
les caractéristiques du bois de Hêtre dépendaient à la fois des facteurs édaphiques et des facteurs génétiques.

POLGE (I973) a souligné d'autre part, l'influence du type de sylviculture et. en particulier, de l'ampleur des houppiers, sur la dureté, la densité et la rétractibilité du bois.

Enfin Polge a donné une définition des paramètres pouvant caractériser un bois de qualité apte au déroulage. Nous rappelons brièvement ces critères : un hêtre de bonne qualité doit être caractérisé par une faible densité, une faible dureté et une faible rétractibilité. Ces critères sont d'ailleurs très fortement corrélés entre eux.

I)'autres critères interviennent bien évidemment, comme la rectitude du fût, ou l'absence de tensions internes pouvant provoquer l'éclatement des billes à l'abattage ou au débit, la présence ou l'absence de cour rouge, etc.

Jans cette note nous n'avons abordé que le problème des deux premiers critères, c'est-à-dire : densité, dureté. Nous avons cherché à préciser, par une enquête générale dans les hêtraies du Nord-Est de la France, quels étaient les facteurs du milieu et certains facteurs sylvicoles qui pouvaient avoir le plus d'importance sur le déterminisme de la qualité du bois de Hêtre.

\section{LES CONDITIONS DE MILIEL}

\section{E'T LES PRINCIPALES CARACTÉRISTIQUES ÉCOLOGIQUES}

\section{DES HÊTRAIES DU NORD-EST DE LA FRANCE}

Nous ne reprendrons pas la description des principaux types de hêtraie; du Nord-Est de la France, qui ont été déjà largement définis antérieurement (LE TAcoN et Timbal, i972; 'Timbal, I974).

Rappelons simplement que notre échantillonnage comprend tous les types de hêtraies du Nord-Fst de la France, depuis 1'Argonne jusqu'aux Hautes-Vosges, mais à l'exclusion des Ardennes, de l'Alsace et du Jura.

Ces hêtraies s'étendent entre $250 \mathrm{~m}$ et I $200 \mathrm{~m}$ d'altitude. Le climat, intermédidiaire entre le climat atlantique et le climat continental en plaine, devient montagnard dans les Vosges (étages bioclimatiques montagnard inférieur, moyen et supérieur).

Les roches-mères sont très variées. Sous ce climat souvent qualifié de "lorrain ", le Hêtre est présent sur tous les substrats (calcaires, grès, granites, limons), à l'exclusion généralement des argiles et des marnes.

Les sols sont tout aussi variés. Tous les types de sols peuvent se rencontrer sous hêtraie sauf ceux à hydromorphie temporaire ou permanente, ou ceux trop fortement podzolisés.

Tous les types d'humus (sauf ceux hydromorphes), existent sous hêtraie, depuis le mull calcique très carbonaté jusqu'au mor. Les mors typiques sont cependant très peu représentés.

Sur le plan phytosociologique, il faut distinguer, d'une part, les hêtraies de l'étage collinéen et montagnard inférieur qui se subdivisent en trois grandes catégories (hêtraies thermoxérophiles du Cephalanthero-Fagion, mésoneutrophiles de l'Eu-Fagion et acidiphiles du Luzulu-Fagion et du Quercion robori-petraeae), et d'autre part, les hêtraies d'altitude de l'Acero-Fagion. 


\section{3. - MÉTHODOLOGIE}

Nous avons choisi 120 placettes dans des futaies de hêtres d'âge variable (de fo à 1 fo ans), de laçon à représenter tous les principaux types de hêtraies par au moins une dizaine d'échantillons.

Dans chaque placette nous avons effectué les mesures ou observations suivantes :

floristiques (relevé phytosociologique) :

pédologiques (description d'un profil de sol avec analyses physiques et chimiques);

-. dendrométriques (âge, hauteur dominante, nombre de tiges, surface terrière. largeur des cernes sur les trois derniers centimètres);

technologiques (couple de torsion et densité).

Rappelons (Polge et Keller, 1972), que la valeur du couple de torsion est très corrélée à la densité et à la dureté du bois (couple de torsion mesuré au torsiomètre).

Sur des fragments de carottes de $5 \mathrm{~cm}$ de longueur, prélevés à la tarière de Presslek, nous avons déterminé la densité du bois par la méthode de saturation intégrale.

Les mesures du couple de torsion et les prélèvements de carottes ont été effectués sur 30 arbres par placette (3 60o carottes au total), entre frévrier et aon̂t 1973.

Seules les mesures de houppiers ont été effectuées sur un nombre beaucoup plus réduit de placettes.

Les liaisons entre ces différents paramètres ont été déterminées par des calculs statistiques simples (analyses de variance, comparaison de moyennes et coefficients de corrélation totaux).

\section{4. — RÉSULTATS}

4. I. - Corrélation entre le couple de torsion et la densité

\section{t. I. I. Corrélation intrapeuplement (fig. I).}
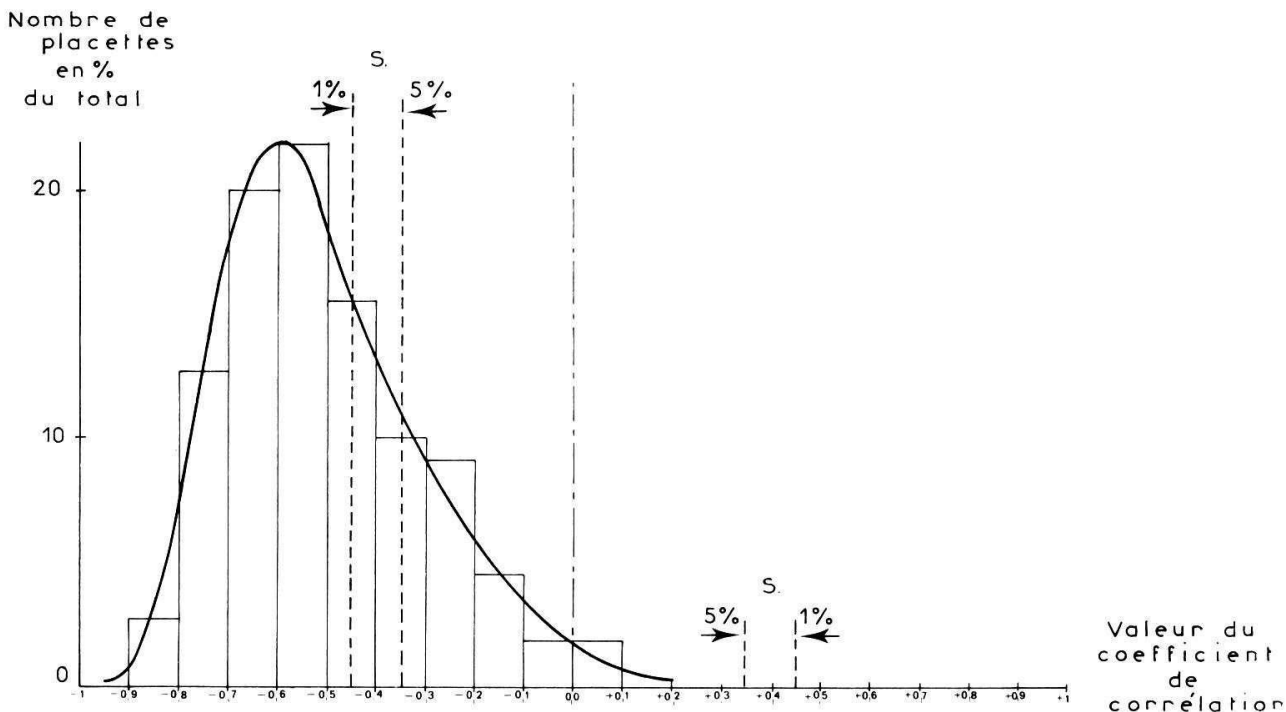

Firi. I. Fréquences relatives des coefficients de corrélation intrapeuplement densite du bois-couple de torsion

Relative frequency of correlation coefficients-wood density.

Torsion torque in beech stands 
La corrélation est très bonne entre le couple de torsion et la densité à l'intérieur des peuplements. Dans 90 p. Ioo de ceux-ci, cette corrélation est positive et significative au seuil de 5 p. Ioo. La valeur du coefficient de corrélation la plus fréquente est comprise entre 0,60 et 0,70 , ce qui est particulièrement élevé ( $r$ à 5 p. IOO $=0,35)$, et peut même atteindre 0,95 .

Ces résultats confirment donc parfaitement l'intérêt de la mesure du couple de torsion (POLGE et KELLER, I970).

\section{I.2. Corrélation interpeuplement.}

Nous avons effectué les mêmes calculs en utilisant cette fois les valeurs moyennes de chaque placette. Entre peuplements, le coefficient de corrélation entre couple de torsion et densité est encore élevé puisqu'il est de 0,495 pour un nombre de degrés de liberté de IOO ( $r$ à 5 p. IOO $=0$, I94 et $r$ à I p. IOO $=0,254)$.

\section{2. - Influence de l'époque de mesure sur la valeur du couple de torsion}

La liaison couple de torsion-densité semblant moins bonne entre peuplements qu'à l'intérieur des peuplements, nous avons cherché à savoir si l'époque de la mesure n'avait pas une influence sur la valeur du couple de torsion. Une partie des mesures a, en effet, été effectuée " hors sève ", et l'autre durant la période de végétation active.

Dans le tableau I, nous avons classé les valeurs du couple de torsion et de la densité suivant trois périodes de prélèvement : mars-avril, juin, et juillet-août.

TABLEAU I

Variation de la densité et du couple de torsion en fonction des dates de prélèvements des carottes

Influence of time of year on wod density variations and torsion torque

\begin{tabular}{|c|c|c|}
\hline Période de mesure & $\begin{array}{l}\text { Valeur moyeme } \\
\text { du couple de torsion } \\
\qquad(\mathrm{cm} / \mathrm{k} g)\end{array}$ & $\begin{array}{l}\text { Valeur movenne } \\
\text { de la densité du boi } \\
\qquad\left(\mathrm{g} / \mathrm{dm}^{3}\right)\end{array}$ \\
\hline Mars-avril $197:$ & $2 \cdot 27,5$ & {$[541,3$} \\
\hline Juin 1973 & 217,9 & 541,9 \\
\hline Juillet-août 1973 & 195,6 & $L_{535,7}$ \\
\hline $\begin{array}{l}\text { Signification au } \\
\text { seuil de } 5 \text { p. } 100\end{array}$ & $\begin{array}{l}\text { Significatif } \\
\text { entre les : périodes }\end{array}$ & Non significatif \\
\hline & & \\
\hline
\end{tabular}

Il n'y a aucune différence significative entre ces trois périodes pour la densité.

Par contre, les différences sont significatives entre ces trois périodes, pour le couple de torsion. Sa valeur est nettement plus faible pendant la période de 
végétation active que "hors sève ", c'est-à-dire durant la période d'arrêt de la végétation.

Si le couple de torsion est globalement très corrélé à la densité, il y a néanmoins une influence de la date de mesure sur la valeur du couple de torsion. Si on reut utiliser ce critère de qualité du bois de hêtre, il est donc nécessaire de mesurer ce couple de torsion à une période bien précise. La période " hors sève " nous semble alors la plus judicieuse, à condition cependant d'éviter les périodes de gel.

Dans ce qui suit, nous n'avons donc utilisé que les mesures d'infradensité, puisqu'elles seules sont indépendantes des périodes de prélèvement des carottes ( ${ }^{1}$.

\section{3. Variations de la densité suivant les régions}

Traditionnellement, les hêtres des plateaux calcaires sont réputés de bonne qualité, alors que ceux des Basses-Vosges par exemple, sur substrat gréseux acide, ont la réputation inverse.

Nous avons donc classé l'ensemble de nos peuplements suivant 5 grandes régions écologiques qui sont d'Ouest en Esst:

- 1'Argonne (Gaize cénomanienne et limons) ;

- les plateaux calcaires (calcaires, argiles de décarbonatation et limons) :

- la plaine lorraine (grès rhétiens, et limons sur argiles ou marnes) :

les Basses-Vosges gréseuses (grès à Voltzias, grès intermédiaires et limons) :

les Vosges moyennes et les Hautes-Vosges (granites et migmatites).

Les valeurs moyennes de la densité pour ces 5 régions sont consignées dans le tableat 2.

TABLEAU 2

lariations de la densité du bois suivant les grande's régions naturelles du Nord-Est de la France

I ariation of wood density according to large natural regions of North-Last of Franee

Régions naturelles

Argonne

Plateaux calcaires

Plaine lorraine

Basses-Vosges

Vosqes moyennes

Hautes-Vosges
Densité movenue ( $\left.\mathrm{g} / \mathrm{dm}^{3}\right)$

$$
\left[\begin{array}{r}
538,4 \\
546,1 \\
538,1 \\
540,5 \\
517,9
\end{array}\right.
$$

V.B. : Les valeurs non différentes significativement au seuıl de .5 p. $10 n$ sont jointes par un trait.

(1) Cette variation de la valeur du couple de torsion en fonction du temps est nette chez les feuillus à feuilles caduques. Elle ne l'est pas pour les résineux. 
Une analyse de variance à un facteur contrôlé, puis une comparaison de moyennes montrent que, seules les Vosges moyennes et les Hautes-Vosges, diffèrent significativement de toutes les autres régions par une densité nettement plus faible.

Toutes les autres régions sont parfaitement semblables. Il n'y a, en particulier aucune différence entre les plateaux calcaires et les Basses-Vosges.

\section{4. - Variations de la densité suivant les massifs forestiers}

Pour déterminer ces variations, nous n'avons conservé que les forêts où les mesures ont été effectuées sur au moins 5 placettes. Les résultats sont consignés dans le tableau 3 .

TABLEAU 3

rariation de la densité moyenne du bois par placette suivant les massifs forestiers (plus de cinq placettes par massif)

Variation of wood density according to different forests

\begin{tabular}{|c|c|c|}
\hline Massif forestier & Région naturelle & $\begin{array}{l}\text { Densité moyemue } \\
\qquad\left(\mathrm{g} / \mathrm{dm}^{3}\right)\end{array}$ \\
\hline L amarche-Morimont & Plaine lorraine & 517,3 \\
\hline Gérardmer & Hautes-Vosges & {$[029,2$} \\
\hline Liffol-le-Grand & Plateaux calcaires & {$[5 \geq 6,6$} \\
\hline Le Corgebin & Plateanx calcaires & {$[539$} \\
\hline Beaulieu & Argonne & $53 x$ \\
\hline Darney & Basses-losges & {$[538$} \\
\hline Evaux & Plateaux calcaires & {$[0,2]$} \\
\hline Auberive & Plateaus calcaires & {$[549$} \\
\hline
\end{tabular}

En considérant les valeurs moyennes prises par la densité, nous pouvons constater que la variabilité est beaucoup plus grande entre forêts qu'entre régions. Par exemple, sur les plateaux calcaires, certaines forêts ont des densités faibles (Liffol-le-Grand) ou fortes (Auberive). De même dans la plaine lorraine qui a une densité moyenne assez élevée $(538, \mathrm{I})$, la forêt de Lamarche a une densité très faible $(5 \mathrm{I} 7,3)$.

Nous allons essayer de voir dans quelle mesure il est possible d'expliquer ces différences entre forêts, soit par les facteurs écologiques du milieu, soit par la sylviculture.

\section{5. - Densité et facteurs écologiques du milien}

\section{5.I. Densité et type d'humus.}

Le type d'humus est une caractéristique stationnelle tout à fait remarquable. Il synthétise l'ensemble des propriétés chimiques, et parfois même physiques, du sol particulièrement en forêt " naturelle ", ce qui est le cas dans les hêtraies du Nord- 
en eau utile. Les mull calciques carbonatés sont en effet caractéristiques des rendzines, dont les réserves en eau sont toujours faibles.

De même, à l'opposé de la gamme des types d'humus, lorsque l'on passe des mull mésotrophes aux mull acides, aux moder puis aux mor (partie droite de la fig. 2), la densité diminue également. Cette diminution va de pair avec celle de la fertilité des sols (dégradation des propriétés chimiques des sols surtout, et, à un moindre degré, diminution des réserves en eau par passage à des substrats de plus en plus sableux).

Sur les humus des stations d'altitude, la densité du bois de Hêtre est faible. Ces stations sont également peu fertiles, mais cette fois pour des raisons climatiques (rigueur du climat, courte période de végétation).

Il apparaît done clairement que la densité du bois du Hêtre est d'autant plus élevée que la station est plus fertile. Tous les facteurs qui diminuent la fertilité stationnelle (facteurs climatiques, physiques ou chimiques), entraînent donc une diminution de la densité du bois de Hêtre.

\section{5.2. Densité et caractéristiques chimiques du sol (fig. 3).}

La réponse de la densité du bois de Hêtre à la fertilité du sol, exprimée suivant un gradient de $\mathrm{pH}$ (type d'humus), étant non linéaire, le calcul des coefficients de corrélation entre les propriétés chimiques du sol et la densité n'a pas de sens si on prend en compte l'ensemble des placettes. Nous avons donc éliminé pour ces calculs, d'une part les hêtraies d'altitude à déterminisme climatique, et, d'autre part, les hêtraies à mull calcique et à mull eutrophe. Les résultats ne sont donc valables que pour la partie droite de la figure 2 , c'est-à-dire pour la partie de la gamme des types d'humus allant du mull mésotrophe au mor.

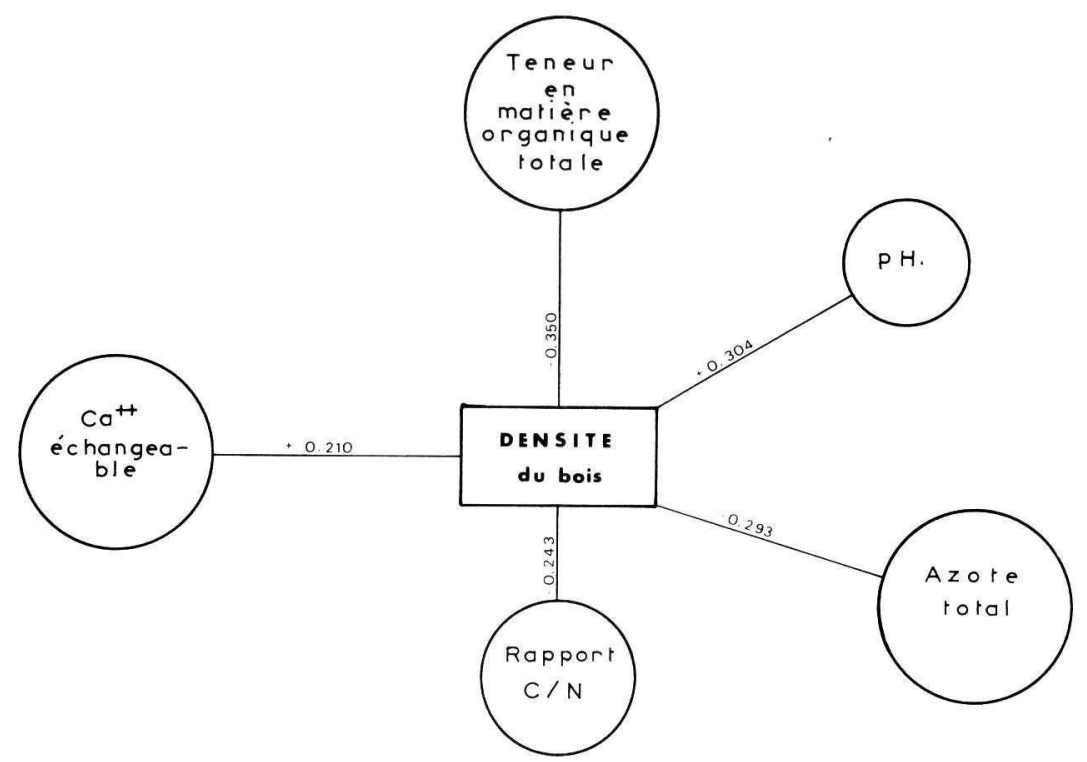

FiG. 3. - Corrélation entre la densité du bois de hêtre et les propriétés chimiques des horizons $A_{1}$ FIG. 3. - Correlation between wood density and chemical properties of $A_{1}$ horizon 
I' 'autre part, nous n'avons pris en compte que les horizons $A_{1}$ et non l'ensemble des caractéristiques chimiques des différents horizons des profils de sol. Il faut en effet rappeler que les caractéristiques de 1'horizon $\mathrm{A}_{1}$ synthétisent généralement très bien les caractéristiques de l'ensemble du profil.

Les résultats des divers calculs sont rassemblés dans la figure 3. On peut y constater que 4 variables sont liées significativement à la densité, dont trois négativement (la teneur en matière organique, l'azote total et le rapport $\mathrm{C} / \mathrm{N}$ ). Une seule variable, le $\mathrm{pH}$, est liée positivement à la densité. Toutes les autres caractéristiques des horizons $A_{1}$ (taux de saturation, calcium échangeable, potassium échangeable et magnésium échangeable et phosphore assimilable), ne semblent pas être liées à la densité. La liaison densité-calcium échangeable est cependant à la limite de la signification.

Les liaisons positives $(\mathrm{pH})$, et négatives (teneur en matière organique totale, rapport $\mathrm{C} / \mathrm{N}$ et teneur en azote total), entre caractéristiques du sol et densité, sont en accord avec les résultats de la figure 2 : lorsque le type d'humus passe du mull au moder, puis au mor, le $\mathrm{pH}$ diminue, le rapport $\mathrm{C} / \mathrm{N}$ augmente également, ainsi que la teneur en matière organique en raison des difficultés de minéralisation. Ces difficultés de minéralisation entraînent une accumulation d'azote total en $A_{1}$, d'où l'existence de cette liaison négative azote total-densité, qui pourrait paraître, a priori, surprenante. Mais cet azote total n'est pas mis à la disposition de l'arbre. La disponibilité en azote minéral est au contraire beaucoup plus faible sur un mor ou un moder que sur un mull, oì la minéralisation est beaucoup plus rapide.

Seule l'absence de liaison entre le taux de saturation et la densité est assez inattendue.

Les calculs effectués avec l'ensemble des mull eutrophes, mésotrophes, acides, des moder et des mor, donnent des résultats identiques, mais les liaisons deviennent plus lâches.

Lorsque l'on effectue les calculs sur la partie gauche de la figure 2 (mull calciques, eutrophes et mésotrophes), les résultats sont évidemment différents. La liaison devient négative entre le $\mathrm{pH}$ et la densité : mais la liaison entre la teneur en matière organique totale et la densité reste négative. En effet, la présence de calcaire entraîne des difficultés de minéralisation de la matière organique humifiée, qui s'accumule dans 1'horizon $\mathrm{A}_{1}$ (mull calcique noir des rendzines).

Ces différents résultats sur les caractéristiques chimiques des horizons $\mathrm{A}_{1}$ des sols confirment donc ceux trouvés par l'étude des liaisons entre types d'humus et densité.

Toutes les caractéristiques du sol qui entraînent une diminution de la fertilité provoquent une diminution de densité et donc une amélioration de la qualité du bois de Hêtre.

\section{6. - Densité et sylviculture}

Rappelons que PoLGE (I973) avait mis en évidence une influence très nette de la sylviculture sur la qualité du bois de Hêtre : Les hêtres à large cime sont moins durs que ceux à cime étroite. Par contre PoLGE avait abouti à la conclusion qu'il ne semblait pas y avoir de liaison entre la largeur des accroissements annuels et la qualité du bois. 
Nous avons essayé de confirmer, ou d'infirmer, ces résultats en étudiant les variations de densité du bois, d'une part à l'intérieur des peuplements, c'est-à-dire des placettes, et, d'autre part, globalement, en classant les peuplements suivant les types de sylviculture pratiquée.

\section{+. 6.I. Variations de la densité à l'intérieur des peuplements.}

\section{6.I. I. Densité et accroissements (fig. 4).}

Pour chacune des 3600 carottes prélevées, nous avons mesuré l'accroissement annuel dans les trois premiers centimètres. Plus exactement, nous l'avons estimé en comptant le nombre de cernes dans les trois premiers centimètres à partir de l'écorce.

Pour chaque placette nous avons calculé le coefficient de corrélation totale (coefficient intrapeuplement), entre la densité et le nombre de cernes dans les trois premiers centimètres. Nous avons reporté sur la figure 4 la valeur de ce coefficient de corrélation en fonction de sa fréquence ramenée en p. Ioo.

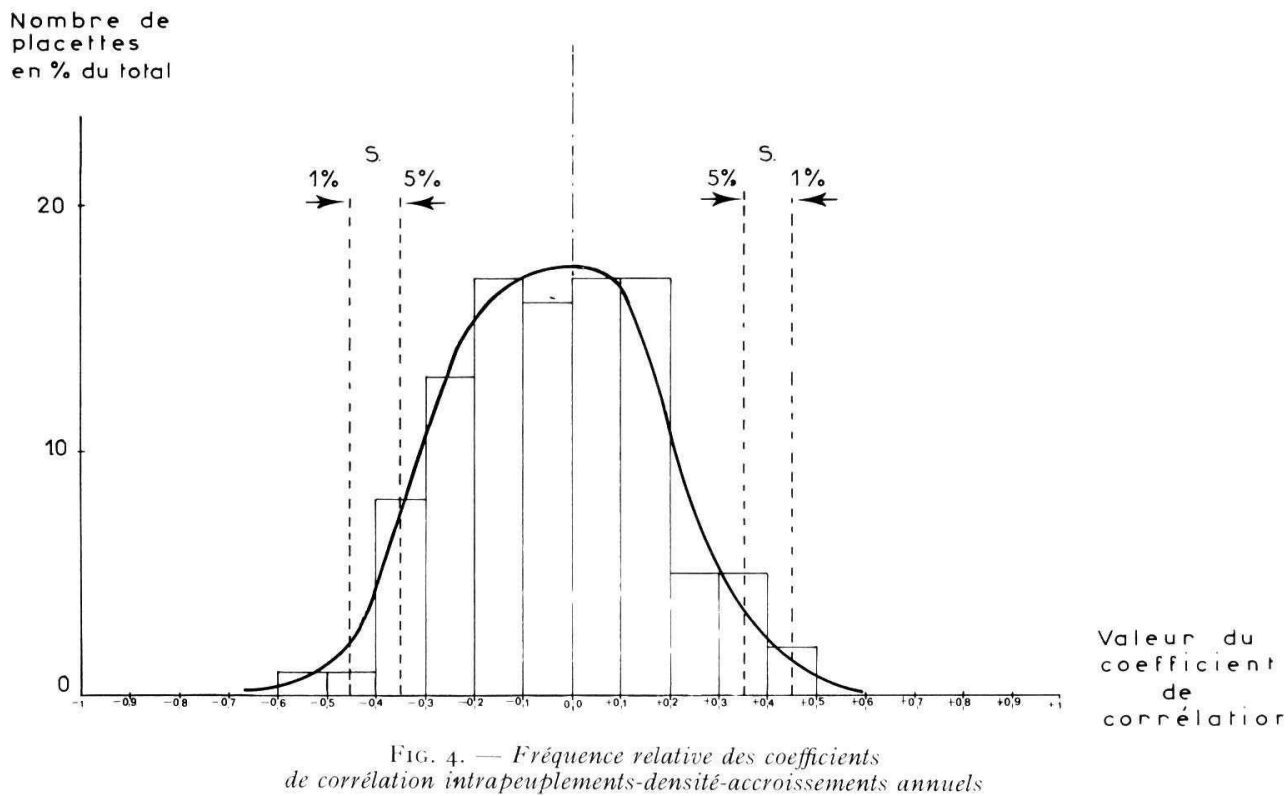

Relative frequency of correlation coefficient between wood density and annual increments

Les résultats sont très clairs : le coefficient de corrélation totale intrapeuplement, entre l'accroissement annuel et la densité du bois est parfaitement centré autour de zéro. Il n'y a donc, à l'intérieur des peuplements, aucune liaison entre accroissement et densité, ce qui confirme, en les généralisant, les résultats de POLGE (I973).

\section{6.I.2. Densité et diamètre (fig. 5).}

Pour chaque placette, nous avons aussi calculé le coefficient de corrélation, entre la densité et le diamètre des tiges.

Ce coefficient de corrélation est toujours négatif comme l'indique clairement 


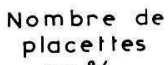

du rotal

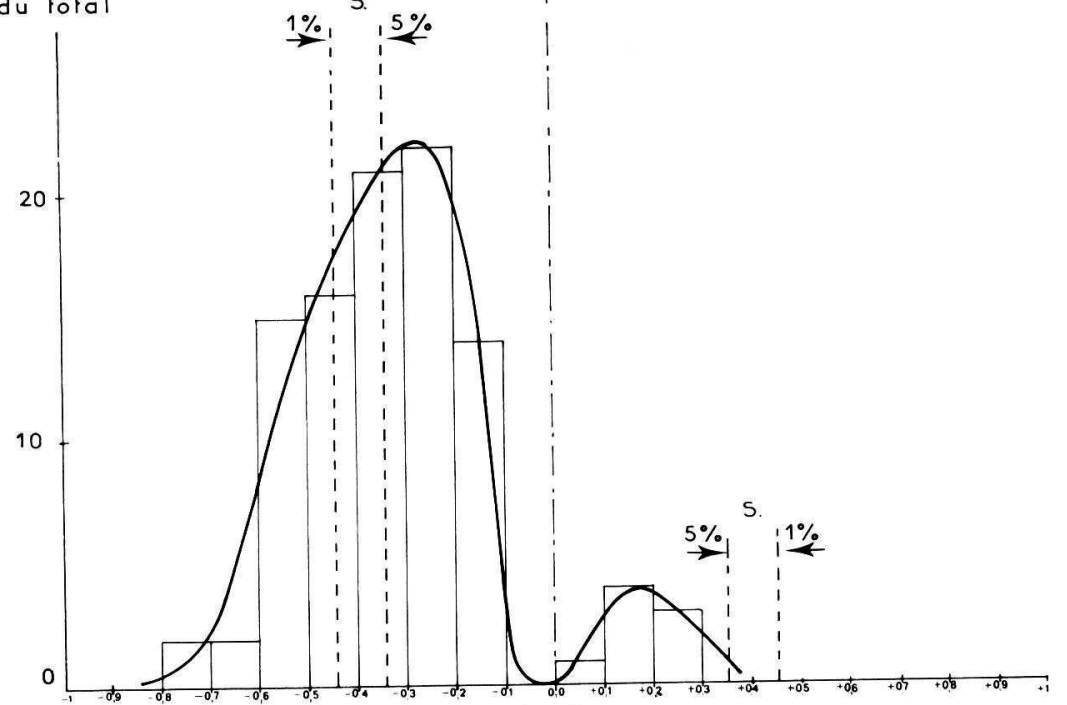

Valeur du coefficient de

FIG. 5. - Fréquence relative des coefficients

de corrélation intrapeuplement densité du bois-diamètre des tiges

Relative frequency of correlation coefficient between wood density and stem diameters

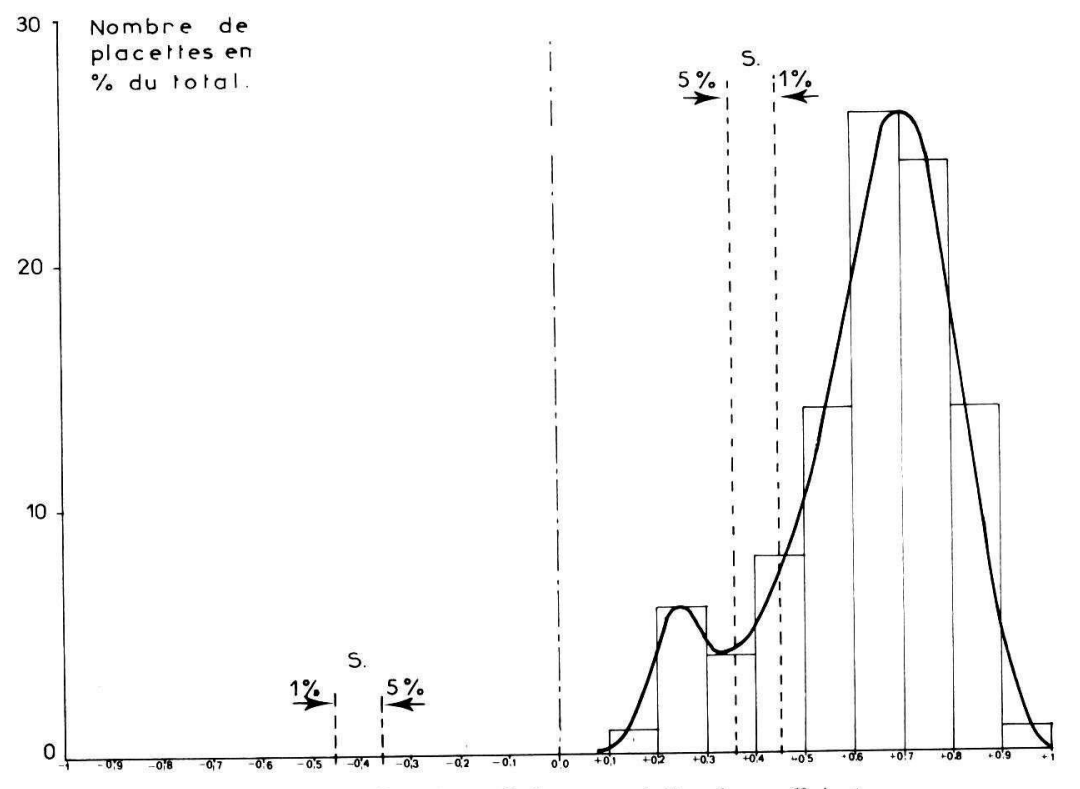

Valeur du coefficient de

Fig. 6. Fréquence relative des coefficients

de corrélation intrapeuplement-diamètre des tiges-nombre de cernes dans les 3 premiers $1 \mathrm{~m}$

Relative frequency of correlation coefficient betreen stem diameters and number of annual rings in the first three centimeters 
la figure 5 . De plus il est significatif pour un nombre important de placettes. Globalement il y a donc bien une liaison entre le diamètre des tiges et la densité. Cette dernière est d'autant plus faible que le diamètre des tiges est plus élevé. Cette liaison est cependant relativement lâche.

\section{6.I.3. Accroissements annuels et diamètre (fig. 6).}

Comme l'indique la figure 6 , la liaison est très bonne entre l'accroissement annuel et le diamètre des tiges. Le coefficient de corrélation intrapeuplement est très significatif pour la très grande majorité des placettes. Il est aussi pratiquement toujours négatif. Le nombre de cernes dans les trois premiers centimètres est donc corrélé négativement avec le diamètre des arbres, ce qui est évidemment parfaitement logique.

\section{6.I.4. Diamètre des tiges et dimension des houppiers.}

Dans trois placettes on a mesuré, suivant huit directions à $45^{\circ}$, les rayons des projections au sol des houppiers, ce qui a permis d'estimer leur surface d'une façon assez précise.

Sur les trois coefficients de corrélation intrapeuplement entre diamètre des arbres et surface des houppiers, un n'est pas significatif, alors que les deux autres sont positifs et significatifs au seuil de I p. I ooo.

Le même calcul portant, cette fois, sur l'ensemble des arbres des trois placettes étudiées, conduit à un coefficient de corrélation totale très significatif de $+0,77 \mathrm{I}$, pour un nombre de degrés de liberté de 80 ( $r$ à I p. IOO $=0,357)$.

Bien que ces calculs ne portent que sur un très petit nombre de placettes, comportant au total 82 arbres, ils montrent qu'il semble bien exister une liaison positive entre la dimension des houppiers et le diamètre des tiges, qui, d'après un certain nombre de résultats partiels déjà acquis, ne peut-être que favorable vis-à-vis de la qualité du bois de Hêtre.

Pour des raisons circonstancielles techniques, la corrélation entre la densité et la dimension des houppiers n'a pu être établie.

\section{6.I.5. Discussion de ces résultats.}

Ces résultats confirment parfaitement les observations de Pol,GE (I972)

La densité est indépendante des accroissements annuels, mais dépend du diamètre des tiges, lui-même corrélé à la dimension des houppiers.

Il est cependant difficile d'expliquer pourquoi la densité du bois diminue lorsque le diamètre des tiges, ou la dimension des houppiers, augmente, alors qu'elle est indépendante de la largeur des accroissements annuels, et que diamètre et accroissements annuels sont très corrélés ( $r=0,77$ significatif à I p. I00).

Seules des analyses plus poussées des composantes de la qualité du bois de Hêtre pourraient, peut-être, apporter une réponse.

Il y a également une certaine contradiction entre l'absence de corrélation intrapeuplement densité-accroissement annuel, et l'existence d'une liaison négative interpeuplement entre la fertilité stationnelle et la densité : plus la station est fertile, plus les accroissements annuels sont larges, et plus la densité est élevée. Or, à l'intérieur d'un peuplement, la densité est indépendante de la largeur des accroissements annuels. 
Cette contradiction peut être en partie levée si l'on admet qu'à l'intérieur d'un peuplement, les variations de la largeur des accroissements annuels, sont surtout en rapport avec la concurrence qu'exercent les arbres entre eux, et donc relativement indépendantes du niveau de fertilité stationnel. Au contraire, entre peuplements, les variations de largeur des cernes sont surtout fonction de la fertilité de la station.

La variation interpeuplement et la variation intrapeuplement de la largeur des cernes seraient donc sous la dépendance de facteurs très différents. Il n'est donc pas étonnant que ces deux types de variations ne soient pas liés de la même façon à la densité. Néanmoins, des études sur les composantes de la densité ne pourraient qu'apporter des informations précieuses sur ce problème complexe.

\section{6.2. Densité et intensité des éclaircies (fig. 7).}

La sylviculture pratiquée dans les I20 peuplements que nous avons étudiés présente une assez grande variation. Sur le graphique de la figure 7 , nous avons porté en abscisse la hauteur dominante des arbres, et en ordonnée, le logarithme du nombre de tiges par hectare.

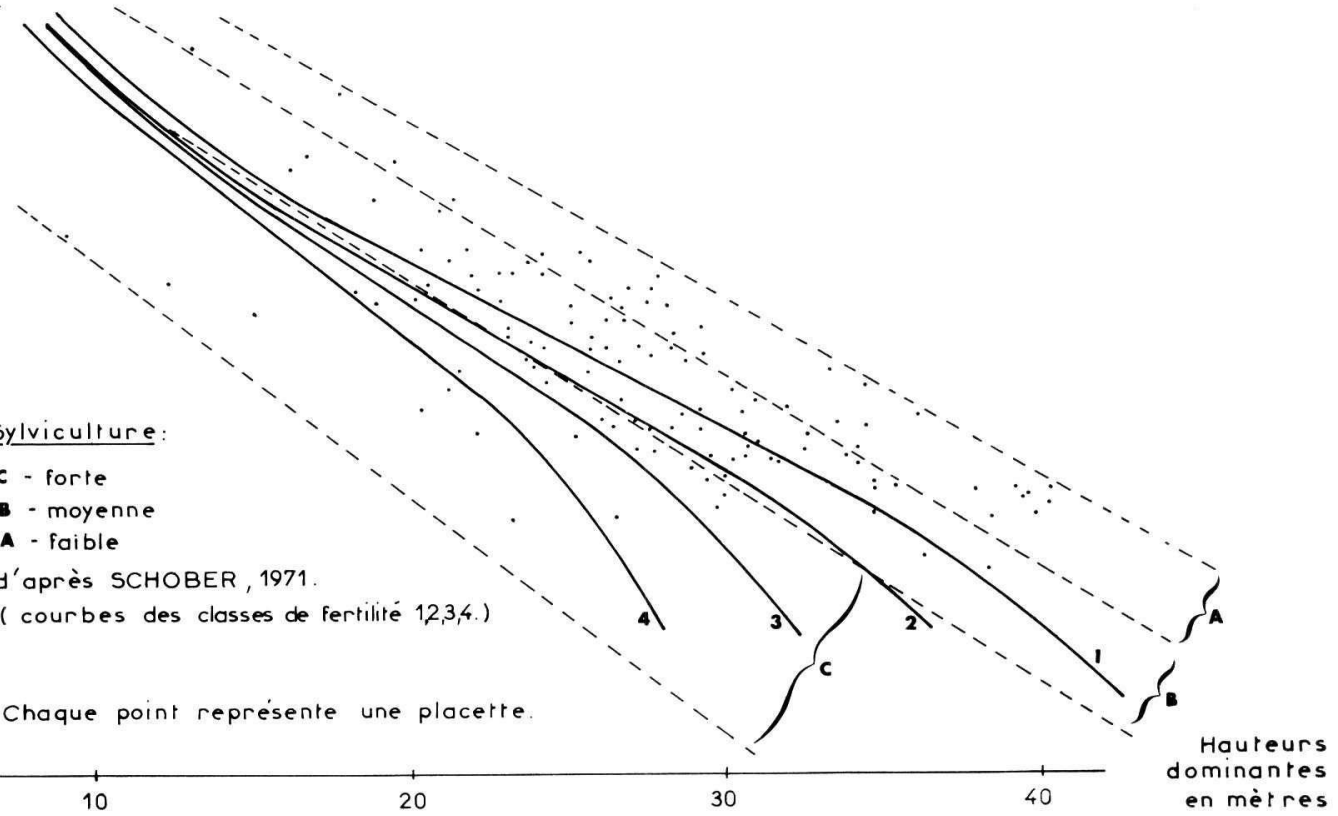

FIG. 7. - Densité du bois et sylviculture

Hood density and thinning intensity

Nous pouvons ainsi constater que la sylviculture pratiquée dans les futaies de Hêtres du Nord-Esst de la France est beaucoup moins intensive que celle pratiquée par exemple en Allemagne. En effet, à hauteur dominante égale, la majorité 
des peuplements que nous avons échantillonnés sont beaucoup plus denses que ceux d'Allemagne comme le montre bien la table de SchoBER à laquelle nous nous sommes référés.

En fonction du nombre de tiges à l'hectare nous avons rangé nos I 20 peuplements du Nord-Est de la France en trois classes d'intensité d'éclaircie (éclaircies fortes, moyennes ou faibles). Pour chaque placette nous avons calculé la densité moyenne globale du bois et la densité moyenne du bois des arbres dominants. Les résultats sont consignés dans le tableau 4 .

\section{TABLEAU 4}

Valeur moyenne de la densité du bois pour tous les arbres (30)

et pour les 5 arbres dominants de chaque placette,

en fonction de l'intensité des éclaircies pratiquées

Wood density of dominant trees and wood density of all trees of sample plots according to thinning intensity

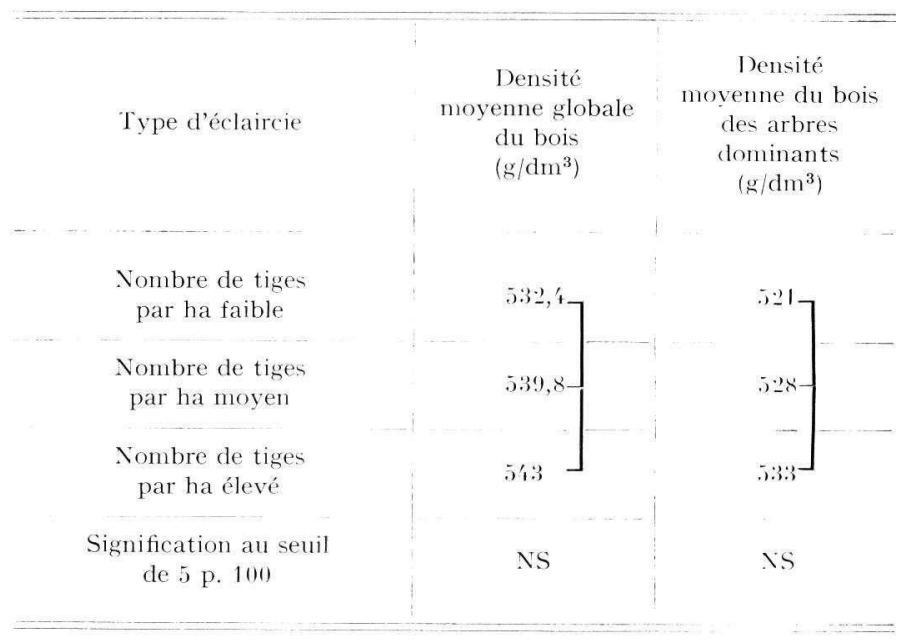

Ils montrent qu'il n'y a pas de différence significative entre les trois types de sylviculture, aussi bien en ce qui concerne la densité moyenne globale que pour la densité moyenne des arbres dominants. Nous pouvons seulement déceler une tendance vers la diminution de la densité lorsque les éclaircies ont été fortes.

On pourrait s'attendre à un effet beaucoup plus net des éclaircies puisque l'augmentation du diamètre des tiges entraîne une diminution de la densité. Deux explications peuvent être avancées. L'effet station est, nous l'avons vu, assez important et peut venir masquer l'effet sylviculture. On peut aussi penser que les éclaircies n'ont pas encore été suffisantes dans les peuplements les moins denses pour entraîner une baisse de l'infradensité par l'intermédiaire de l'effet des houppiers. 
L'influence des éclaircies ne pourra être correctement appréciée qu'à l'aide de dispositifs spécialement conçus à cet effet. Une expérience mise en place par la Station de Sylviculture et Production du C.N.R.F. en forêt de Souilly (55) pourra bientôt être exploitée. D'autre part, la comparaison de la densité du bois de Hêtre, entre taillis-sous-futaie et futaie, pourra permettre d'apprécier l'effet diamètre des houppiers.

\section{5. - CONCLUSIONS}

Cette étude de la valeur du couple de torsion et de la densité du bois de Hêtre dans r 20 placettes de futaie du Nord-Est de la France, confirme les résultats précédemment acquis par POLGE (I972), et apporte un certain nombre d'éléments noureaux.

La liaison entre le couple de torsion et la densité est très bonne à condition que les mesures du couple de torsion soient effectuées à une même période de l'année. Il apparaît en effet que la valeur du couple de torsion est plus élevée pendant la période d'arrêt de la végétation que pendant la période de végétation active.

L'indépendance de la densité envers la largeur des accroissements annuels se trouve confirmée, ainsi que la diminution de la densité avec le diamètre des tiges et la dimension des houppiers.

Néanmoins, nous ne pouvons mettre en évidence un effet sylviculture ni sur la densité moyenne du bois des peuplements ni sur la densité moyenne des arbres dominants. Les hêtraies échantillonnées ne semblent donc pas avoir été suffisamment éclaircies, et il n'y a pas, dans notre échantillonnage, de peuplement suffisamment éclairci, pour que l'effet diamètre des tiges, ou dimension des houppiers, puisse être décelé.

L'effet station sur la densité du bois peut être, par contre, facilement mis en évidence. La densité est d'autant plus élevée que les stations sont plus fertiles. Tous les facteurs qui diminuent la fertilité stationnelle, que ce soit des facteurs climatiques (hêtraies d'altitude), des facteurs chimiques (hêtraies à mor ou à moder), ou des facteurs physiques (hêtraies calcicoles xérophiles sur rendzine) diminuent la densité du bois. Par exemple, lorsque le type d'humus passe du mull mésotrophe au mor, la densité diminue et apparaît être liée positivement à la baisse du $\mathrm{pH}$, et négativement, au rapport $\mathrm{C} / \mathrm{N}$.

Cette augmentation de la densité du bois avec le niveau de fertilité des stations est en contradiction avec l'absence de corrélation intrapeuplement entre la densité du bois et la largeur des accroissements annuels, et avee la corrélation négative intrapeuplement entre la densité et le diamètre des tiges. Néanmoins, les variations d'accroissement annuel inter et intrapeuplement sont sous la dépendance de facteurs très différents. Les premières dépendent de la fertilité des stations, tandis que les secondes dépendent de la concurrence entre les individus. Il n'est donc pas surprenant que ces deux types de variations n'agissent pas de la même manière sur la densité du bois.

La comparaison de la densité du bois suivant les grandes régions naturelles du Nord-Est de la France montre que, seules, les Hautes-Vosges, diffèrent des autres 
régions par une densité du bois plus faible. I1 n'y a, en particulier, pas de différence significative à cet égard, entre la hêtraie des Basses-Vosges, qui a une mauvaise réputation de qualité chez les utilisateurs, et les hêtraies des plateaux calcaires.

Nous pouvons peut-être nous demander si cette bonne réputation des Hêtres des plateaux calcaires n'est pas une conséquence du traitement en taillis-sousfutaie qui est encore la règle dans beaucoup de forêts de cette région (taillis-sousfutaie vrai ou taillis-sous-futaie vieilli du fait d'une politique de conversion en futaie régulière), et qui favorise le développement des houppiers et des gros diamètres. Dans les Basses-Vosges, au contraire, les hêtraies sont traditionnellement traitées en futaies régulières denses, ce qui fournit des cimes étroites et de faibles diamètres. Quand le même traitement de futaie régulière dense est appliqué sur les plateaux calcaires, la qualité du bois, du moins en ce qui concerne les critères de dureté, de densité et de rétractibilité, ne semble plus différer de celle des Basses-Vosges. Cette hypothèse pourra ou non être validée, lorsque la même étude aura pu être menée à bien en taillis sous futaie. Le traitement en futaie régulière n'est d'ailleurs pas incompatible avec la production d'arbres à cime développée, si des interventions dynamiques en éclaircie sont faites dans des peuplements jeunes.

Enfin, certaines forêts présentent des densités faibles, sans que l'on puisse invoquer un effet station ou un effet sylviculture. Ce seraient alors les facteurs génétiques qu'il faudrait chercher et l'on pourrait donc parler de l'existence de "crus de Hêtre "), comme cela se fait déjà pour le Chêne.

Ces résultats ne sont évidemment que partiels, et seront complétés en particulier en tenant compte d'autres critères importants de la qualité du bois de Hêtre, comme les phénomènes de tension interne, et de rétractibilité.

Reçu pour publication en décembre 1975

\author{
SUMMARY \\ DENSITY OF BEECH WOOD IN THE NORTH-EAST OF FRANCE. \\ INFLUENCE OF THE ENVIRONMENTAL CHARACTERISTICS \\ AND OF THE TYPE OF SILVICULTURE
}

This work has been carried out on I20 sample-plots of beech high-forest, of variable ages in the North-East of France. These sample-plots have been selected in order to get all the ccological variability of the country. In each sample-plot, the torsion torque was measured, on thirty trees, and a test piece of wood was collected, on which the annual increment and the wood density were calculated.

The relationship between wood density and the torsion torque is satisfactory when the measures were effected at the same time in the year.

Site effect is very clear on wood density. All the ecological factors which reduce site fertility, reduce wood density too.

The silvicultural effect is less easy to analyse. Indeed, the calculated correlations are not significant and the sampled stands have not been thinned enough in order to prove a silvicultural effect. 


\section{ZUSAMMENFASSUNG}

\section{DIE HOLZQUALITÄT DER BUCHE IN ABHÄNGIGKEIT VON STANDORT UND WALDBAULICHER BEHANDLUNG}

Die Untersuchung wurde in I 20 Buchenhochwald - Probaflächen verschiedenen Alters in Nordostfrankreich unternommen. Die Probeflächen wurden derart gewählt dass sie die Vielfalt der ökologischen Verhältnisse dieser Region weitgehend repräsentieren.

In jeder Probefläche wurde an 30 Bäumen Drehmomentmessungen mit einem Torsiometer ausgeführt und je ein Bohrspan entnommen. An jedem Bohrspan wurde die Anzahl der Jahrringe der aüsseren $30 \mathrm{Mm}$ gezählt und an einem $50 \mathrm{Mm}$ langen Stück wurde die Rohdichte bestimmt. Anschliessend wurden die Korrelationsskoeffizienten zwischen diesen verschiedenen Merkmalen berechnet.

Die Beziehung zwischen Rohdichte und Drehmoment ist sehr gut, wenn die Messungen zu gleicher Jahreszeit erfolgen.

Im Gegensatz dazu wurde festgestellt, dass zwischen Rohdichte und Jahrringbreite keine Beziehung besteht.

Die Rohdichte nimmt auch gleichlaufend mit Durchmesser und Kronendimensionen ab.

Es zeigt sich ein sehr deutlicher Einfluss des Standortes auf die Rohdichte des Buchenholzes. Alle ökologischen Faktoren, die die Fertilität des Standortes verringern, führen gleichfalls zu einer Abnahme der Rodichte.

Der Einfluss des waldbaulichen Behandlung ist sehr schwer zu interpretieren. Die Berechneten Korrelationskoeffizienten sind nicht signifikant aber es scheint, dass die untersuchten Probedächen meist nur sehr schwach durchforstet wurden und daher ungenügend differenziert. so dass der Finfluss der waldbaulichen Behandlung nicht herausgestellt werden kann.

\section{RÉFÉRENCES BIBLIOGRAPHIQUES}

Becker M., I968. Le Hêtre (Fagus silvatica L.) et ses problèmes en forêt de Villers-Cotterêt (o2). Contribution à la mise au point d'une méthode dynamique d'étude écologique du milieu forestier. Doc. C.N.R.F. à diff. limit., I $968 / 3$.

Le Tacon F., Trmbal J., 1972. A propos des conditions écologiques des hêtraies du Nord-list et du Nord-Ouest de la France. Rev. forest. Fr., 24 (3), i $87-200$.

L. Tacon F., Timbal J., I973. Valeurs indicatrices des principales espèces végétales des hètraies du Nord-Est de la France, vis-à-vis du type d'humus. Rev. forest. Fr., 25 (4), 269-282.

PiCARD J. F., i970. Les forêts sur Rhétien dans le département des Vosges. Nouvelle contribution à la mise au point d'une méthode dynamique d'étude phyto-écologique du milieu forestier. Thèse de Doctorat de $3^{e}$ cycle, Nancy.

Polgf H., I973. Etat actuel des recherches sur la qualité du bois de Hêtre. Bull. Techn. O....F., 4.

Polge H., Keller R., i97o. Première appréciation de la qualité du bois en forêt par utilisation d'un torsiomètre. Ann. Sci. forest., 27 (2), p. 197.

Polge H., Keller R., Thiercelin F., ig72. Effets du sol et de l'hérédité sur la croissance et les caractéristiques anatomiques de jeunes plants de Hêtre. Doc. C.N.R.F. à diff. limit., I972/I.

Schober R., 1967. Yield and thinning of beech woods in Germany. Ann. Acad. Ital. Sci. forest., 16, 31 7$35 \mathrm{I}$.

Schoble R., I971. Dif. Rotbuchi, J. 1). Sauerlander's Verlag, Frankfurt an Main. 